\section{Psoriasis-Arthritis: Hürden bei der Implementierung von Treat to target}

\author{
Dures E et al. Mixed methods study of clinicians' \\ perspectives on barriers to implementation of \\ treat to target in psoriatic arthritis. Ann Rheum \\ Dis 2020; 79: 1031-1036
}

Bei sogenannten Treat-to-Target-Ansätzen (T2T) werden bestimmte Therapieziele definiert und Therapiefortschritte durch regelmäßige Kontrollen überprüft. Wenn nötig, können Behandlungen individuell angepasst werden. Bei der Psoriasis-Arthritis (PsA) verbessert T2T klinische und Patienten-berichtete Outcomes. Obwohl es in europäischen Leitlinien empfohlen wird, wird T2T bei der Behandlung von PsA selten angewendet. Eine neue Studie untersucht Barrieren der Implementierung.

Im Rahmen der Studie wurde medizinisches Personal befragt, das in die Behandlung von Patienten mit PsA involviert ist. In einem qualitativen Teil wurden die Teilnehmer per Telefon oder in persönlichen Fokusgruppen interviewt. Mithilfe der Erkenntnisse aus den Gesprächen wurde eine quantitative Umfrage erstellt, die eine größere Gruppe an Rheumatologen schriftlich beantworten sollte. Diese Ergebnisse wurden dann statistisch-deskriptiv ausgewertet.
Zehn rheumatologisch-tätige Personen aus dem Vereinten Königreich nahmen an den Telefoninterviews teil (acht Rheumatologen, ein Physiotherapeut, ein spezialisierter Krankenpfleger). Deren Arbeitserfahrung betrug zwischen 5 und 26 Jahren, die Interviews dauerten im Mittel 30 Min. An den persönlichen Fokusgruppen nahmen sieben Rheumatologen und 2 andere medizinische Mitarbeiter aus dem Vereinten Königreich teil. Deren Arbeitserfahrung reichte von 3-22 Jahren. Die Fokusgruppe dauerte $102 \mathrm{Min}$.

In den persönlichen Interviews zeigte sich, dass folgende Aspekte einer Implementierung von T2T bei PsA im Weg stehen:

- Komplexität (Differenzialdiagnose PsA vs. Rheumatoide Arthritis, Parameter zur Erfassung der Krankheitsaktivität, Erfassung der Beschwerden, Ressourcen (v. a. zeitlich, personell))

- Schwierigkeit von Änderungen der aktuellen Praxis (Zurückhaltung aufgrund organisatorischer Faktoren, unterschiedliche individuelle Entschlossenheit, Änderungen vorzunehmen)

In der schriftlichen Umfrage wurden 153 in der Rheumatologie-tätige Personen näher zu diesen Aspekten befragt, 138 davon waren Rheumatologen (der Rest andere Fachärzte, Assistenzärzte). Deren Berufserfahrung reichte von einem bis 50 Jahren (Median 16 Jahre, Mittel 18,5 Jahre). Die Teilnehmer stammten aus dem Vereinten Königreich (33), anderen europäischen Ländern (37), Nordamerika (21), Südamerika (25), Asien-Pazifik (19) und anderen Regionen (7). Die Auswertung der Umfrage erbrachte u. a. folgende Ergebnisse:

- 54,5\% der Befragten halten begrenzte Zeit für regelmäßige Termine mit einer Erfassung von Therapiezielen für ein großes Hindernis bei der Implementierung von T2T bei PsA

- $35 \%$ halten mangelnde Kenntnisse bei der Beurteilung von Hauterkrankungen für ein Hindernis

- $40 \%$ stimmten der Aussage zu, dass ein T2T-Ansatz nicht flexibel genug ist, um eine individuell passende Behandlung zu finden
- $15 \%$ gaben an nicht zu glauben, dass ein T2T-Ansatz das Outcome der Patienten verbessert

Die Teilnehmer wurden auch gefragt, welche Faktoren eine Implementierung von T2T erleichtern könnten. Dabei zeigten sich folgende Ergebnisse:

- Bereitstellung eines Protokolls (86,4\%)

- Eine Person, die die Einführung und Umsetzung vor Ort koordiniert $(80,9 \%)$

- (Personelle) Unterstützung des Teams, das die Therapieerfolge untersuchen soll $(83,3 \%)$

- Schulungen in T2T (69,8\%)

Auch regelmäßige Audits, Feedbackgespräche und die Verfügbarkeit elektronischer Datenbanken wurden als Faktoren identifiziert, die eine Implementierung erleichtern könnten.

\section{FAZIT}

In der klinischen Praxis werden Patienten mit Psoriasis-Arthritis trotz Empfehlungen nicht immer mit Treat to target-Ansätzen behandelt. Die vorliegende Umfrage unter Rheumatologen zeigt, dass eine Implementierung mit einfachen Maßnahmen wie der Bereitstellung von Protokollen und Schulungen erleichtert werden könnte. Auch ist einigen Rheumatologen nicht bewusst, dass T2T-Ansätze bei PsA nachweislich das Outcome der Patienten verbessert.

Marisa Kurz M. Sc. B. A. München 